\title{
Search for high-mass resonances decaying into leptonic final states using the ATLAS detector
}

\author{
Yosuke Takubo* on behalf of the ATLAS collaboration \\ High Energy Accelerator Research Organization (KEK), 1-1 Oho Tsukuba Ibaraki, 305-0801, \\ Japan \\ E-mail: yosuke.takubo@kek.jp
}

\begin{abstract}
Many theories beyond the Standard Model predict new phenomena with the final states of well isolated high- $p_{\mathrm{T}}$ leptons. Searches for new physics with these signatures are performed in the ATLAS experiment at the LHC. The ATLAS experiment collected $139 \mathrm{fb}^{-1}$ of data with $13 \mathrm{TeV}$ $p p$ colliding energy during 2015 to 2018 (Run 2), that is available for physics analyses. In this article, the results to search for a heavy particle decaying into leptonic final states with full Run 2 dataset as well as the combination analysis and prospects for HL-LHC are reported.
\end{abstract}

European Physical Society Conference on High Energy Physics - EPS-HEP2019 -

10-17 July, 2019

Ghent, Belgium

${ }^{*}$ Speaker. 


\section{Introduction}

The resonance search is the most standard way to find a new particle. These searches in the ATLAS experiment are motivated by many new physics models beyond the Standard Model (SM) aiming to resolve the hierarchy problem such as the Randall-Sundrum (RS) model with a warped extra dimension [1], extended Higgs sectors as in the two Higgs-doublet model [2], composite Higgs bosons [3] or extended gauge sectors as in Grand Unified Theories [4, 5, 6].

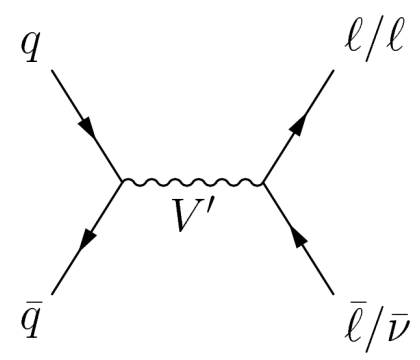

Figure 1: Feynman diagram for resonance production mediated by a new heavy boson $\left(V^{\prime}=W^{\prime}\right.$ or $\left.Z^{\prime}\right)$ in Drell-Yan mechanism, that decays into $\ell v$ or $\ell \ell$.

The proton-proton $(p p)$ colliding energy at Large Hadron Collider (LHC) was increased from $8 \mathrm{TeV}$ to $13 \mathrm{TeV}$ after long shutdown 1 in 2013 to 2014. The ATLAS experiment at LHC collected $139 \mathrm{fb}^{-1}$ of data during 2015 to 2018 (Run 2), that is available for physics analyses. Searches for new heavy spin-1 bosons ( $W^{\prime}$ and $\left.Z^{\prime}\right)$ are performed by using data taken in Run 2, that are produced by Drell-Yan (DY) mechanisms as shown in Figure 1. In this article, the analysis results of searches for $W^{\prime}$ and $Z^{\prime}$ in the leptonic final states with full Run 2 dataset [7, 8], the combination analysis with $36.1 \mathrm{fb}^{-1}$ of data [9] and prospect at High-Luminosity LHC (HL-LHC) [10] are described.

\section{2. $W^{\prime} \rightarrow \ell v$ search}

The interpretation in this search uses the Sequential Standard Model (SSM) [11] as the benchmark, in which the $W^{\prime}$ boson $\left(W_{\mathrm{SSM}}^{\prime}\right)$ couples to fermions with the same strength as the $W$ boson in the SM but with suppressed coupling to SM bosons. The dominant background source in this search originates from DY production of $W$ bosons. Discrimination between signal and background events relies on the transverse mass $\left(m_{\mathrm{T}}\right)$ computed from the charged-lepton transverse momentum $\left(p_{\mathrm{T}}\right)$ and the missing transverse momentum (whose magnitude is denoted as $E_{\mathrm{T}}^{\mathrm{miss}}$ ) in the event:

$$
m_{\mathrm{T}}=\sqrt{2 p_{\mathrm{T}} E_{\mathrm{T}}^{\mathrm{miss}}\left(1-\cos \phi_{\ell v}\right)}
$$

where $\phi_{\ell v}$ is the angle between the charged lepton and missing transverse momentum directions in the transverse plane. Figure 2 shows the transverse mass distributions in the electron and muon channels for events passing the full selection. No significant excess was observed in both cases, i.e., the distributions are consistent with the SM expectation.

Figure 3 shows observed and expected upper limits at the 95\% confidence level (CL) on the $p p \rightarrow W^{\prime} \rightarrow \ell v$ cross section in the combination of electron and muon channels as a function of $W_{\text {SSM }}^{\prime}$ mass with the full signal line shape from the SSM, where the interference between the $W^{\prime}$ 

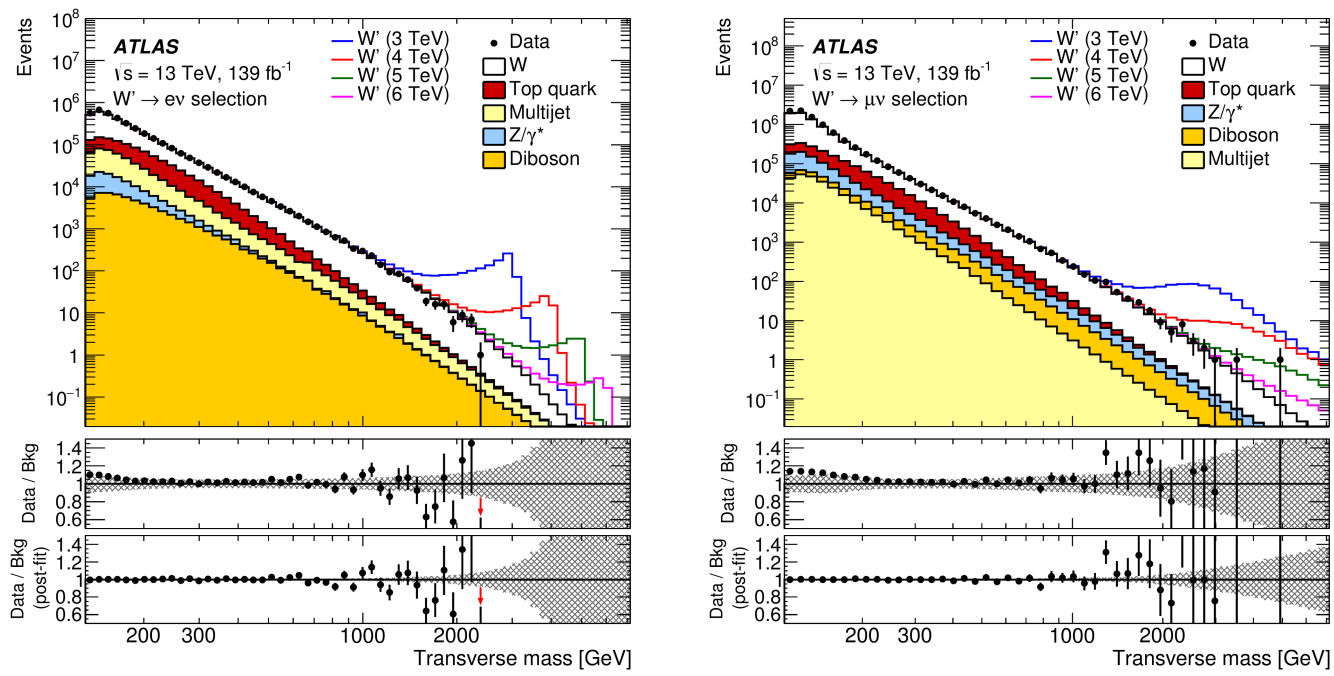

Figure 2: Distributions of the transverse mass for data and predicted background events in the electron (left) and muon (right) channels [7]. Expected signal distributions for several $W_{\text {SSM }}^{\prime}$ boson masses are shown stacked on top of the total expected background. The middle panels show ratios of the number of events observed in the data to the expected total background count, while the lower panels show the same ratio when taking into account the pulls on the nuisance parameters observed in the statistical analysis. The hatched bands represent the total uncertainty in the background estimate. Arrows in the middle and lower panels for the electron channel indicate data points that lie outside the vertical axis range.

signal and the SM DY background is not taken into account. The crossing point of the upper limit and the model cross-section curve gives the limit on $W_{\text {SSM }}^{\prime}$ mass, summarizing the obtained mass limits in Table 1.

\begin{tabular}{ccc}
\hline \hline & \multicolumn{2}{c}{$m\left(W^{\prime}\right)$ lower limit $[\mathrm{TeV}]$} \\
Decay & Observed & Expected \\
\hline$W^{\prime} \rightarrow e v$ & 6.0 & 5.7 \\
$W^{\prime} \rightarrow \mu \nu$ & 5.1 & 5.1 \\
\hline$W^{\prime} \rightarrow \ell \nu$ & 6.0 & 5.8 \\
\hline \hline
\end{tabular}

Table 1: Observed and expected 95\% CL lower limits on the $W_{\text {SSM }}^{\prime}$ mass in the electron and muon channels and their combination [7].

\section{3. $Z^{\prime} \rightarrow \ell \ell$ search}

In this search, $Z^{\prime}$ defined in $\operatorname{SSM}\left(Z_{\mathrm{SSM}}^{\prime}\right), \mathrm{E}_{6}$-motivated Grand Unification model $\left(Z_{\chi}^{\prime}\right.$ and $\left.Z_{\psi}^{\prime}\right)$ [12] and the Heavy Vector Triplet (HVT) model ( $Z_{\mathrm{HVT}}^{\prime}$ ) [13] are assumed as the benchmark. In the first two models, the $Z^{\prime}$ boson is a singlet, associated with a new U(1) gauge group, and generally its couplings to the SM $W$ and $Z$ bosons are assumed to be zero. $Z_{\mathrm{SSM}}^{\prime}$ boson has the same fermion couplings as the $\mathrm{SM} Z$ boson.

The $Z_{\mathrm{HVT}}^{\prime}$ boson is a neutral member of a new $\mathrm{SU}(2)$ gauge group, i.e., part of a triplet and exists with two new charged heavy bosons $\left(W_{\mathrm{HVT}}^{\prime}\right)$ with which it is nearly degenerate in mass. The 


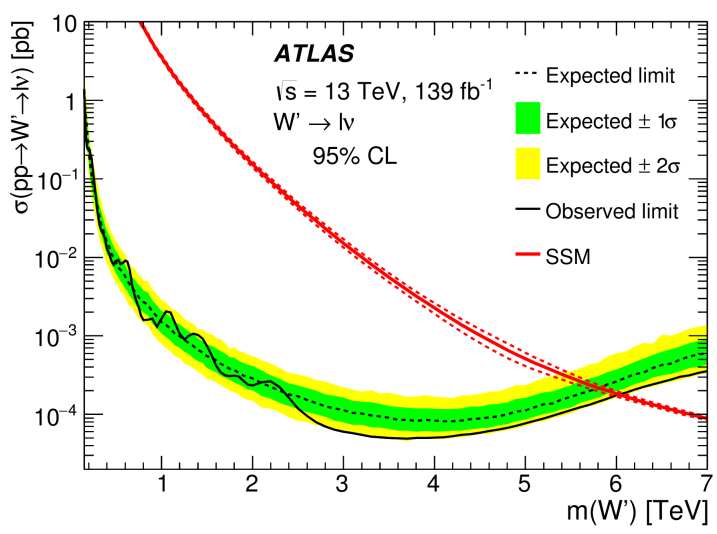

Figure 3: Observed and expected upper limits at the 95\% CL on the $p p \rightarrow W^{\prime} \rightarrow \ell v$ cross section in the combination of electron and muon channels as a function of $W_{\mathrm{SSM}}^{\prime}$ mass [7]. The dashed lines surrounding the SSM cross-section curve (solid line) correspond to the combination of PDF, $\alpha_{\mathbb{s}}$, renormalization, and factorization scale uncertainties (for illustration only).

HVT model provides generic framework with couplings of $W^{\prime}$ and $Z^{\prime}$ to SM fermions and Higgs. For that reason, this model is used to evaluate constraint on the couplings.

Figure 4 shows distributions of the dielectron and dimuon invariant mass for events passing the full selection. A resonant signal is searched for by fitting the distributions. The fit function consists of a generic signal shape and a smooth functional form for the background. The dilepton mass distributions are consistent with the smoothly falling background expectation, and no significant excess is observed.
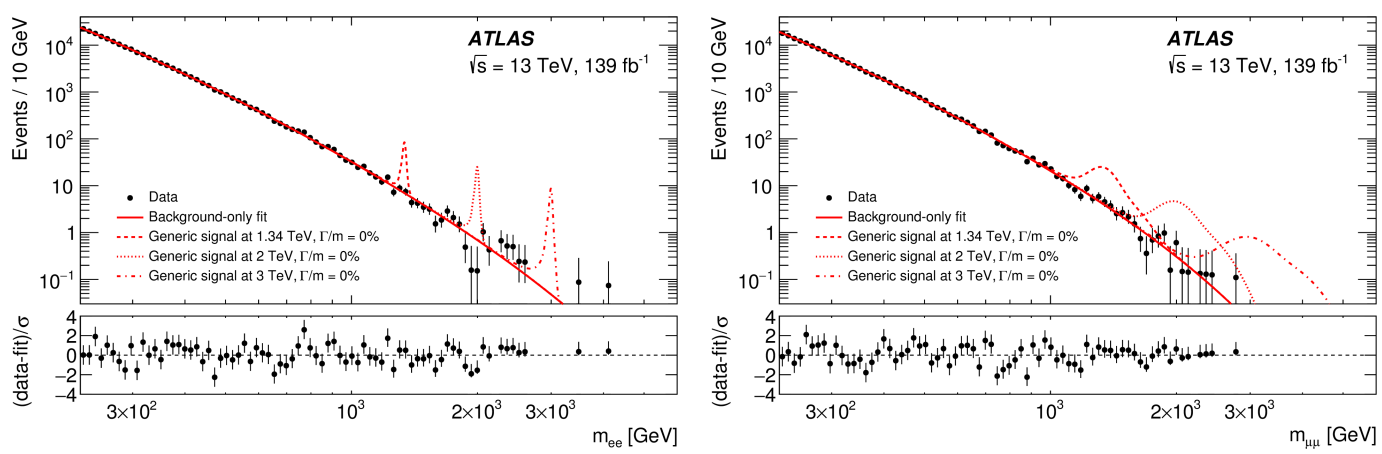

Figure 4: Distribution of the invariant mass for events passing the full selection in electron (left) and muon (right) channels [8]. Generic zero-width signal shapes, scaled to 20 times the value of the corresponding expected upper limit at 95\% CL on the fiducial cross-section times branching ratio, with pole masses of $m_{\chi}$ $=1.34,2$ and $3 \mathrm{TeV}$ as well as background-only fits are superimposed. The data points are plotted at the centre of each bin. The error bars indicate statistical uncertainties only. The differences between the data and the fit results in units of standard deviations of the statistical uncertainty are shown in the bottom panels.

Figure 5 shows the upper limits at 95\% CL on the fiducial cross-section times branching ratio as a function of pole mass for different mass widths for the combined dilepton channel. The generic cross-section limits at $\Gamma / m=0.5 \%, 1.2 \%$ and $3.0 \%$ are compared with the model predictions of 
$Z_{\psi}^{\prime}, Z_{\chi}^{\prime}$ and $Z_{\mathrm{SSM}}^{\prime}$, respectively, to evaluate the mass limits that are summarized in Table 2.
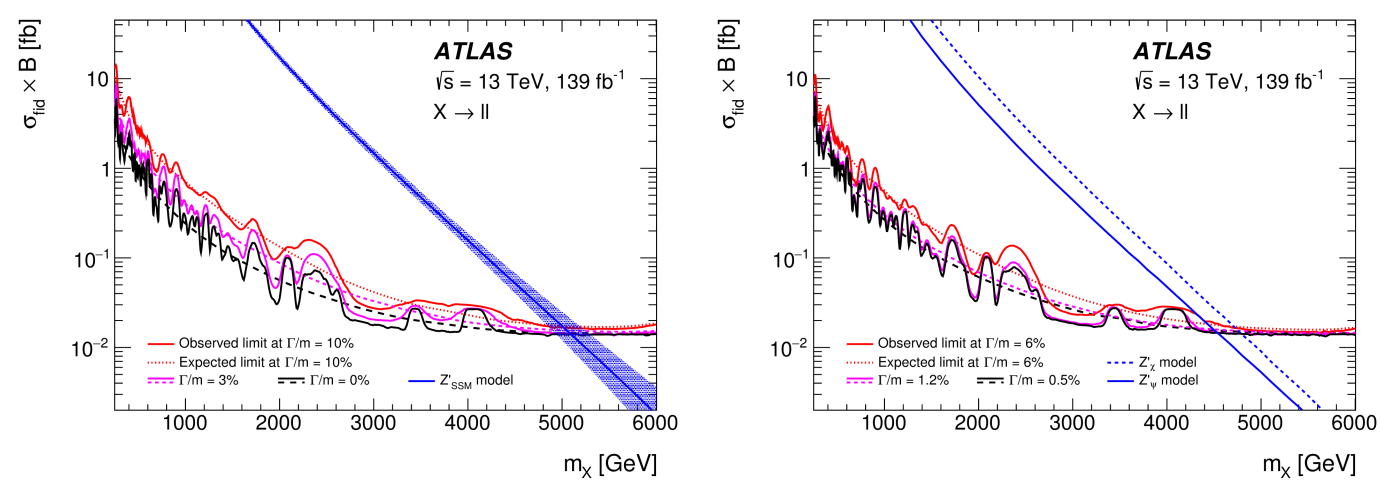

Figure 5: Upper limits at 95\% CL on the fiducial cross-section times branching ratio as a function of pole mass for the zero-width, 3\%, 10\% (left) and $0.5 \%, 1.2 \%, 6 \%$ (right) relative width signals for the combined dilepton channel [8]. Observed limits are shown as solid lines and expected limits as dotted/dashed lines. Also shown are theoretical cross-sections for $Z_{\mathrm{SSM}}^{\prime}(\Gamma / m=3.0 \%)$ (left) and $Z_{\chi}^{\prime}(\Gamma / \mathrm{m}=1.2 \%)$ and $Z_{\psi}^{\prime}(\Gamma / \mathrm{m}=0.5 \%)$ (right) in the fiducial region. The signal theoretical uncertainties are shown as a band on the $Z_{\mathrm{SSM}}^{\prime}$ theory line and are derived as in Ref. [14]. They are shown for illustration purposes, and are not included in the limit calculation.

\begin{tabular}{l|cc|cc|cc}
\hline \multirow{2}{*}{ Model } & \multicolumn{3}{|c}{ Lower limits on $m_{Z^{\prime}}[\mathrm{TeV}]$} \\
& obs & exp & \multicolumn{2}{c}{$\mu \mu$} & \multicolumn{2}{c}{$\ell \ell$} \\
& obs & exp & obs & exp \\
\hline$Z_{\psi}^{\prime}$ & 4.1 & 4.3 & 4.0 & 4.0 & 4.5 & 4.5 \\
$Z_{\chi}^{\prime}$ & 4.6 & 4.6 & 4.2 & 4.2 & 4.8 & 4.8 \\
$Z_{\text {SSM }}^{\prime}$ & 4.9 & 4.9 & 4.5 & 4.5 & 5.1 & 5.1 \\
\hline
\end{tabular}

Table 2: Observed and expected 95\% CL lower limits on $m_{Z^{\prime}}$ for three $Z^{\prime}$ gauge boson models, quoted to the nearest $100 \mathrm{GeV}$ in the $e e$ and $\mu \mu$ channels as well as their combination $(\ell, \ell)$ [8].

The cross-section limits are converted into exclusion contours in coupling space of the HVT model. Figure 6 shows observed 95\% exclusion contours in the HVT parameter space of $\left(g_{h}, g_{f}\right)$ with $g_{f} \equiv g_{\ell}=g_{q}$ and $\left(g_{q}, g_{\ell}\right)$ with $g_{h}=0$ for resonance masses of 3, 4 and $5 \mathrm{TeV}$ for the dilepton channel, where $g_{\ell}, g_{q}$ and $g_{h}$ correspond to the coupling strengths of the triplet field to lepton, quark and Higgs and vector-boson fields, respectively.

\section{Combination analysis}

The combination analysis was performed by using $36.1 \mathrm{fb}^{-1}$ of data taken in Run 2, including the $V V$ channels $W Z \rightarrow q q q q, \ell v q q, \ell v \ell \ell ; W W \rightarrow q q q q, \ell v q q, \ell v \ell v$; and $Z Z \rightarrow q q q q, v v q q, \ell \ell q q$, $\ell \ell v v, \ell \ell \ell \ell$ and the $V H$ channels $W H \rightarrow q q b b, \ell v b b$ and $Z H \rightarrow q q b b, v v b b$, $\ell \ell b b$, as well as the lepton-antilepton channels $\ell v$ and $\ell \ell$. The charged leptons $\ell$ are either electrons or muons. For the $V V$ and $V H$ decay channels involving leptonic decays of vector bosons, $\tau$-leptons are included as part of the signal since $\tau$-lepton decays into electrons or muons provide a small amount of additional acceptance. 

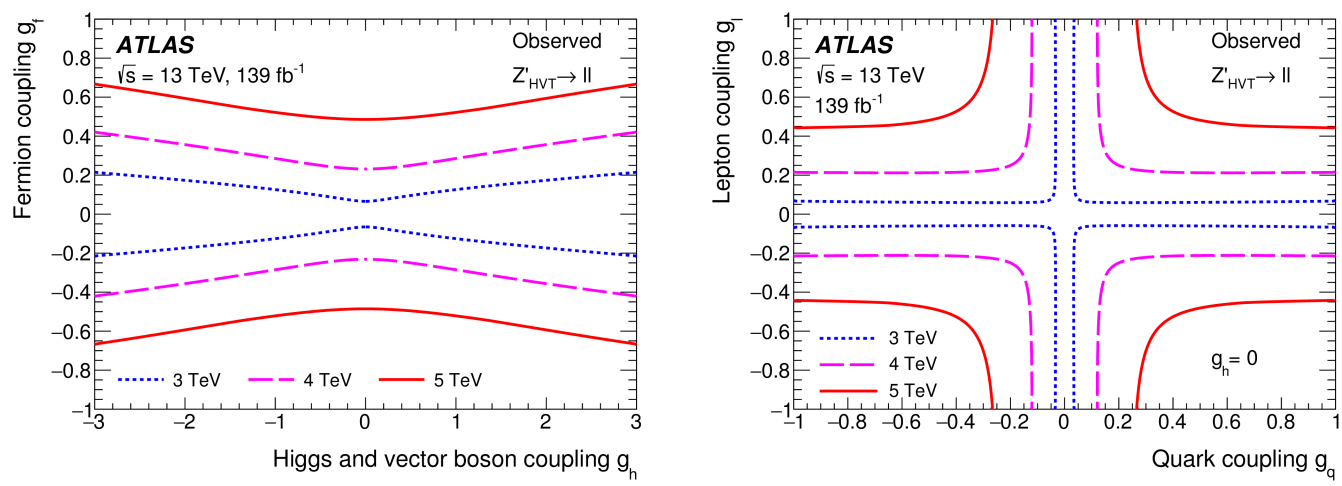

Figure 6: Observed 95\% exclusion contours in the HVT parameter space in $\left(g_{h}, g_{f}\right)$ with $g_{f} \equiv g_{\ell}=g_{q}$ (left) and $\left(g_{q}, g_{\ell}\right)$ with $g_{h}$ set to zero (right), for resonance masses of 3, 4 and $5 \mathrm{TeV}$ for the dilepton channel [8]. The area outside the curves is excluded.

No significant deviation from the SM predictions is observed, therefore, the cross-section limits are evaluated in terms of constraints on couplings of the heavy vector-boson triplet to quarks $\left(g_{q}\right)$, leptons $\left(g_{f}\right)$ and Higgs boson $\left(g_{H}\right)$ in context of the HVT model. Figure 7 shows observed 95\% CL exclusion contours in the HVT parameter space of $\left(g_{H}, g_{f}\right)$ and $\left(g_{q}, g_{\ell}\right)$ for resonances of mass 3, 4 and $5 \mathrm{TeV}$ for the combination of $V V, V H$ and $\ell v / \ell \ell$ channels. A and B in the plots represent two scenarios with different relative strength of $g_{H}$ and $g_{f}$. A denotes $g_{H}=-0.56$ and $g_{f}=g_{q}=g_{\ell}=-0.55$, reproducing the phenomenology of weakly coupled models. B implements a strongly coupled scenario with $g_{H}=-2.9$ and $g_{f}=0.14$. The leptonic final states $(\ell v$ and $\ell \ell$ ) provide the strongest constraint on the couplings. The remarkable point is that the combination analysis with $36.1 \mathrm{fb}^{-1}$ gives stronger constraint on the coupling than those in $Z^{\prime} \rightarrow \ell \ell$ only analysis with $139 \mathrm{fb}^{-1}$ that are shown in Figure 6.
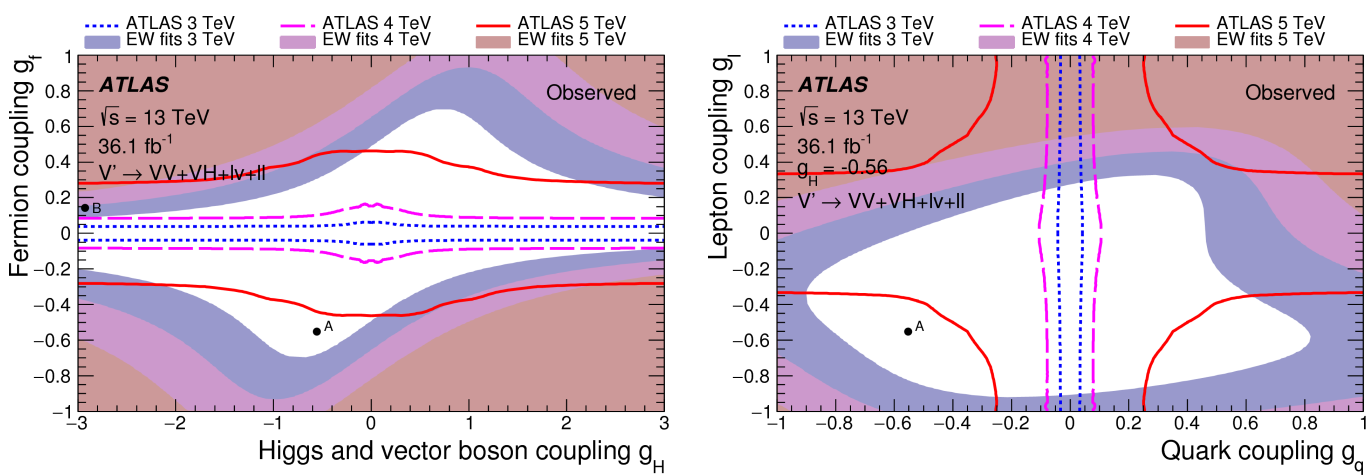

Figure 7: Observed 95\% CL exclusion contours in the HVT parameter space in $\left(g_{H}, g_{f}\right)$ (left) and $\left(g_{q}, g_{\ell}\right)$ (right) for resonances of mass 3,4 and $5 \mathrm{TeV}$ for the combination of $V V, V H$, and $\ell v / \ell \ell$ channels [9]. The areas outside the curves are excluded, as are the filled regions which show the constraints from precision electroweak measurements.

\section{5. $V^{\prime} \rightarrow \ell \ell / \ell v$ search at HL-LHC}

LHC will be upgraded to HL-LHC after 2026 with five times larger instantaneous luminosity 
$\left(5 \times 10^{34} \mathrm{~cm}^{-2} \mathrm{~s}^{-1}\right)$ or more, compared to the LHC design value $\left(1 \times 10^{34} \mathrm{~cm}^{-2} \mathrm{~s}^{-1}\right)$. Search for heavy resonances in the leptonic final states will be performed at HL-LHC.

Figure 8 shows the expected upper limits on cross-section times branching ratio $(\sigma \times \mathrm{BR})$ as a function of the $W_{\text {SSM }}^{\prime}$ (left) and $Z_{\psi}^{\prime}$ (right) boson mass in the combined electron and muon channels for $14 \mathrm{TeV} p p$ colliding energy, assuming $3000 \mathrm{fb}^{-1}$ of data. The sensitivity to $W^{\prime}$ and $Z^{\prime}$ masses will be improved by up to $2 \mathrm{TeV}$, compared to the results with a full Run 2 dataset. Given that, the resonance searches with the leptonic final states remain to be important at HL-LHC.
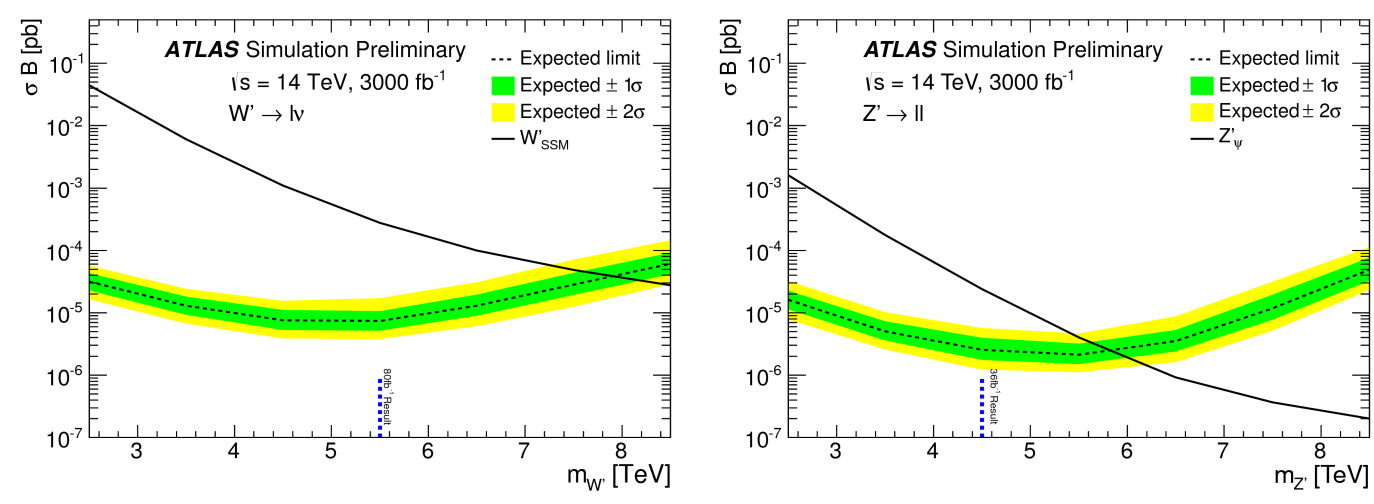

Figure 8: Expected (dashed black line) upper limits on cross-section times branching ratio $(\sigma \times \mathrm{BR})$ as a function of the $W_{\text {SSM }}^{\prime}$ (left) and $Z_{\psi}^{\prime}$ (right) boson mass in the combined electron and muon channels for $\sqrt{s}=$ $14 \mathrm{TeV}$ collisions and an integrated luminosity value of $3000 \mathrm{fb}^{-1}$ [10]. The $1 \sigma$ (green) and $2 \sigma$ (yellow) expected limit bands are also shown. The predicted $\sigma \times \mathrm{BR}$ for $W_{\mathrm{SSM}}^{\prime}$ (left) and $Z_{\psi}^{\prime}$ (right) production is shown as a black line. The blue marker shows the limits obtained with the Run 2 analysis with $36 \mathrm{fb}^{-1}$ of data.

\section{Summary and conclusions}

Searches for high mass resonances with the leptonic final states are important physics program in the ATLAS experiment. The analyses were performed by using $139 \mathrm{fb}^{-1}$ of a full Run2 dataset, and the sensitivity to $W^{\prime}$ and $Z^{\prime}$ is significantly improved, compared to the previous results with $36.1 \mathrm{fb}^{-1}$. The combination analysis including the final states of $\ell v$ and $\ell \ell$ as well as $V V$ and $V H$ was performed with $36.1 \mathrm{fb}^{-1}$ of data. The results give stronger constraint on the couplings of $W^{\prime}$ and $Z^{\prime}$ to SM particles than $Z^{\prime}$ only analysis with $139 \mathrm{fb}^{-1}$ of a full Run2 dataset.

Improvement of the mass limit up to $2 \mathrm{TeV}$ is expected with much larger statistics in HLLHC. For that reason, searches for high-mass resonances with the leptonic final states remain to be important program also in HL-LHC.

\section{References}

[1] L. Randall and R. Sundrum, Large Mass Hierarchy from a Small Extra Dimension, Phys. Rev. Lett. 83, 3370 (1999).

[2] G. C. Branco, P. M. Ferreira, L. Lavoura, M. N. Rebelo, M. Sher, and J. P. Silva, Theory and phenomenology of two-Higgs-doublet models, Phys. Rep. 516, 1 (2012). 
[3] R. Contino, D. Marzocca, D. Pappadopulo, and R. Rattazzi, On the effect of resonances in composite Higgs phenomenology, J. High Energy Phys. 10 (2011) 081.

[4] J. C. Pati and A. Salam, Lepton number as the fourth color, Phys. Rev. D 10, 275 (1974); Erratum, Phys. Rev. D11, 703 (E) (1975).

[5] H. Georgi and S. Glashow, Unity of All Elementary-Particle Forces, Phys. Rev. Lett. 32, 438 (1974).

[6] H. Fritzsch and P. Minkowski, Unified interactions of leptons and hadrons, Ann. Phys. (N. Y.) 93, 193 (1975).

[7] ATLAS Collaboration, Search for a heavy charged boson in events with a charged lepton and missing transverse momentum from pp collisions at $\sqrt{s}=13 \mathrm{TeV}$ with the ATLAS detector, Phys. Rev. D 100, 052013 (2019), arXiv:1906.05609 [hep-ex].

[8] ATLAS Collaboration, Search for high-mass dilepton resonances using $139 \mathrm{fb}^{1}$ of pp collision data collected at $\sqrt{s}=13 \mathrm{TeV}$ with the ATLAS detector, Phys. Lett. B 796 (2019) 68, arXiv:1903.06248 [hep-ex].

[9] ATLAS Collaboration, Combination of searches for heavy resonances decaying into bosonic and leptonic final states using $36 \mathrm{fb}^{-1}$ of proton-proton collision data at $\sqrt{s}=13 \mathrm{TeV}$ with the ATLAS detector, Phys. Rev. D 98, 052008 (2018), arXiv:1808.02380 [hep-ex].

[10] ATLAS Collaboration, Prospects for searches for heavy $Z$ and $W$ bosons in fermionic final states with the ATLAS experiment at the HL-LHC, ATLAS-CONF-2011-068, https://cds.cern.ch/record/2650549.

[11] G. Altarelli, B. Mele, and M. Ruiz-Altaba, Search for new heavy vector bosons in $p \bar{p}$ colliders, Z. Phys. C 45 (1989) 109, Erratum: Z. Phys. C 47 (1990) 676.

[12] D. London and J. L. Rosner, Extra gauge bosons in E(6), Phys. Rev. D 34 (1986) 1530.

[13] F. del Aguila, J. de Blas and M. Perez-Victoria, Electroweak limits on general new vector bosons, JHEP 09 (2010) 033, arXiv: 1005.3998 [hep-ph].

[14] ATLAS Collaboration, Search for new high-mass phenomena in the dilepton final state using $36 \mathrm{fb}^{-1}$ of proton-proton collision data at $\sqrt{s}=13 \mathrm{TeV}$ with the ATLAS detector, JHEP 10 (2017) 182, arXiv:1707.02424 [hep-ex]. 